\title{
Primary Nonadherence, Associated Clinical Outcomes, and Health Care Resource Use Among Patients with Rheumatoid Arthritis Prescribed Treatment with Injectable Biologic Disease-Modifying Antirheumatic Drugs
}

\author{
James Harnett, PharmD, MS; Daniel Wiederkehr, BS; Robert Gerber, PharmD; David Gruben, PhD; \\ Jeffrey Bourret, PharmD; and Andrew Koenig, DO
}

\begin{abstract}
BACKGROUND: Adherence to biologic disease-modifying antirheumatic drugs (bDMARDs) among patients with rheumatoid arthritis (RA) is often suboptimal in routine clinical practice. Low or nonadherence can reduce the effectiveness of bDMARD therapies.
\end{abstract}

OBJECTIVE: To evaluate filling of newly prescribed initial bDMARDs for the treatment of RA and evaluate potential for characterizing treatment decisions and patient outcomes.

METHODS: In this retrospective cohort analysis, patients aged $\geq 18$ years with an RA diagnosis (ICD-9-CM code 714.xx) were selected from a de-identified database of clinical information from the Electronic Health Record (EHR; Humedica) database linked to health care claims (Optum) from commercial and Medicare Advantage health plans (2007-2013). The first biologic prescription date in EHR was the index date. Patients were categorized as filling the prescription within 30 days (early fillers), 31-180 days (late fillers), or not at all within 180 days (nonfillers) of index date.

RESULTS: Of 373 patients meeting inclusion criteria, 170 (45.6\%), 59 (15.8\%), and $144(38.6 \%)$ were categorized as early fillers, late fillers, and nonfillers, respectively. Most prescriptions were written or ordered for tumor necrosis factor inhibitors (88.7\%). Compared with late and nonfillers, early fillers were younger and more likely to be female, with higher pain scores (among those reporting pain scores) and RA severity scores pre-index, and filled more prescriptions for any reason pre-index. More nonfillers (66.0\%) were Medicare patients than early (17.7\%) and late (35.6\%) fillers. During days 0-30 post-index, conventional synthetic DMARD use was greatest for early fillers (45.9\%) and lowest among nonfillers (24.3\%); however, during days 31-180 post-index, the proportion was highest for late fillers $(61.0 \%)$ and lowest for nonfillers (35.4\%). Of early fillers, $12.9 \%$ did not fill/receive a bDMARD after 30 days. Only 23 patients had pre/post-index pain scores, and 47 patients had a rationale for stopping or not filling a bDMARD. In patients with pharmacy and medical coverage for 180 days post-index, early fillers had greater RA-related pharmacy and medical resource use and costs than late and nonfillers combined.

CONCLUSIONS: These findings confirm a high rate of primary nonadherence to bDMARDs among patients with RA.

J Manag Care Spec Pharm. 2016;22(3):209-18

Copyright $\odot 2016$, Academy of Managed Care Pharmacy. All rights reserved.

\section{What is already known about this subject}

Adherence is a general concern for pharmacotherapy; in rheumatoid arthritis (RA), adherence to disease-modifying antirheumatic drugs (DMARDs) is often suboptimal.

Low adherence or nonadherence can reduce the effectiveness of any therapy, including DMARD therapies, leading to suboptimal treatment response, delayed recovery, disease progression, and the need for more aggressive or additive treatments with the potential for adverse events.

Nonadherence rates among patients receiving DMARDs in RA typically range from $41 \%$ to $75 \%$.

\section{What this study adds}

More than $50 \%$ of patients with RA who were prescribed injectable biologic DMARDS (bDMARDs; 88.7\% tumor necrosis factor inhibitors) did not fill the bDMARD prescription via a pharmacy or receive a bDMARD administration in outpatient/inpatient settings within 30 days of the index prescription; by 180 days post-index, more than $40 \%$ of patients had not yet filled or received a prescription for a bDMARD.

Health care resource use and costs were highest for patients who filled a prescription or received a bDMARD within 30 days of the index prescription during short-term observation.

$\mathrm{R}$ heumatoid arthritis (RA) is a chronic and debilitating autoimmune disease characterized by persistent synovitis, systemic inflammation, and joint destruction in most patients, particularly those undertreated or untreated. Early diagnosis and effective treatment with disease-modifying antirheumatic drugs (DMARDs) is important in improving treatment outcomes. ${ }^{1}$

Although conventional synthetic DMARDs (csDMARDs; e.g., methotrexate, sulfasalazine, hydroxychloroquine, and leflunomide) remain the cornerstone of RA pharmacotherapy, biologic DMARDs (bDMARDs) have revolutionized treatment in recent years, improving signs and symptoms and slowing/ inhibiting structural damage with a well-characterized safety 
profile. ${ }^{2}$ Because bDMARDs cost substantially more than csDMARDs, ${ }^{3}$ they are recommended by the American College of Rheumatology (ACR) and the European League Against Rheumatism (EULAR) for use in patients who have previously failed csDMARD treatment. bDMARDs are infused or injected (by syringe or prefilled auto-injector) as monotherapy or with csDMARDs. ${ }^{4,5}$ bDMARDs can be used as first-line therapy in DMARD-naive patients with highly aggressive, erosive disease, ${ }^{4,5}$ or in patients for whom csDMARDs are contraindicated.

Five classes of bDMARDs are available for the treatment of RA. Tumor necrosis factor inhibitors (TNFi) constitute the largest group, which includes etanercept, infliximab, adalimumab, certolizumab pegol, and golimumab. bDMARDs with alternative mechanisms of action include anakinra (interleukin [IL]-1 receptor antagonist), tocilizumab (IL-6 receptor antagonist), abatacept (cytotoxic T lymphocyte antigen [CTLA]-4 immunoglobulin), and rituximab (anti-CD20 B cell antagonist).

Adherence to chronic therapy is often suboptimal in clinical practice, ${ }^{6,7}$ with the World Health Organization reporting that $<50 \%$ of patients take medications as prescribed. ${ }^{8}$ In addition, up to $30 \%$ of prescriptions in the United States are never filled, ${ }^{9}$ costing the health care system $\$ 100$ billion to $\$ 300$ billion annually. ${ }^{10,11}$ Low adherence or nonadherence can reduce the effectiveness of any therapy, including bDMARD therapies, leading to suboptimal treatment response, delayed recovery, disease progression, and the need for more aggressive or additive treatments with the potential for adverse events. ${ }^{12}$ Frequently, health care providers are not given full disclosure when the patient reduces the dose, which may lead to unnecessary exposure to co-medications or dose/frequency adjustments.

Several factors influence patient adherence to therapy, including patient-related factors (e.g., age, sex, education, and socioeconomic status), therapy-related factors (e.g., efficacy, tolerability, convenience, mode/frequency of administration, and costs), and physician-related factors (e.g., disease knowledge and relationship with the medical team). ${ }^{13}$ Furthermore, patient beliefs about treatment have been shown to influence adherence to medication. A recent review by Horne et al. (2013) reported that interventions to optimize adherence were more likely to be effective if they considered patients' beliefs about their illness and treatment and how they assess their need for the medication relative to concerns about the potential adverse effects of taking it. ${ }^{14}$

Medication nonadherence can be categorized as primary nonadherence, which occurs when a patient does not fill the index prescription, or secondary nonadherence, which occurs when a patient discontinues therapy after filling the index prescription. ${ }^{15}$ Although several studies have used U.S. prescription claims data to specifically evaluate nonadherence to bDMARDs among patients with $\mathrm{RA},{ }^{16-19}$ these analyses evaluated secondary nonadherence rather than primary nonadherence.
The purpose of this exploratory analysis was to evaluate the filling of newly prescribed initial bDMARD therapies for RA and to characterize patient outcomes in a real-world setting.

\section{Methods}

\section{Study Design}

This was an exploratory, retrospective cohort analysis of deidentified data from the Humedica Electronic Health Record (EHR) database linked to health care claims (Optum) from commercial and Medicare Advantage health plans during 2007-2013. Linked data were required to evaluate prescriptions by the physician and subsequent filling via a pharmacy, as well as in-office administrations. This linked dataset provides an objective assessment of gaps in treatment patterns.

EHR Database. The longitudinal clinical repository is derived from multiple large health care provider organizations treating patients throughout the United States. EHR data are aggregated directly from providers and include multiple EHRs across the continuum of care, both inpatient and ambulatory, to provide a comprehensive clinical overview that includes medications, laboratory results, vital signs, physician notes, diagnoses, procedures, demographics, hospitalizations, and outpatient visits. Once aggregated, data are normalized, validated, and de-identified. The data are certified as de-identified by an independent statistical expert following the statistical de-identification rules of the Health Insurance Portability and Accountability Act (HIPAA) and managed according to customer data use agreements. ${ }^{20,21}$

Humedica data files contain 2 broad classes of information relevant to the current analysis: unstructured data extracted from physician, pathology, and radiology notes and clinical reports, and structured (coded) data, consisting of demographic, clinical, and operational facts. Terms are extracted from unstructured data using proprietary software that conducts a context-sensitive search for researcher-specified terms and modifiers in a similar manner to the Mayo Clinic cTAKES system. Structured items and natural language processing (NLP)-derived terms are associated with patient records and observation dates, allowing longitudinal, within-patient tracking. The NLP system uses vocabulary from the Unified Medical Language System, which includes the Logical Observation Identifiers Names and Codes, Systemized Nomenclature of Medicine-Clinical Terms, and RxNorm.

Patient-reported pain scores are recorded during discrete health care provider interactions. Providers may use different methods, including the Visual Analogue Scale $(100 \mathrm{~mm}$ horizontal line anchored by the terms no pain $[0 \mathrm{~mm}-4 \mathrm{~mm}]$, mild pain [ $5 \mathrm{~mm}-44 \mathrm{~mm}$ ], moderate pain [ $45 \mathrm{~mm}-74 \mathrm{~mm}]$, and severe pain $[75 \mathrm{~mm}-100 \mathrm{~mm}])$ and the Wong-Baker FACES Scale (0-5 rating, where 0 indicates no pain and 1-5 indicates increasing intensities of pain), to solicit patient measures. Pain 
scores may be recorded in structured data elements of EHR or harvested from notes using NLP.

Claims. Claims data were linked by Optum for a subset of patients in the Humedica EHR who were members of Optum's affiliate national insurer. The Optum DataMart is an integrated database consisting of enrollment, inpatient and outpatient medical claims, pharmaceutical claims, and laboratory results. Optum's integrated claims-clinical dataset is statistically deidentified under the Expert Determination method consistent with HIPAA and managed according to customer data use agreements. ${ }^{20,21}$ The integrated dataset leverages a proprietary Optum algorithm that uses both salting and cryptographic hashing.

\section{Patients}

Eligible patients were aged $\geq 18$ years (at index) with $\geq 2$ outpatient visits or 1 inpatient diagnosis of RA (International Classification of Diseases, Ninth Revision, Clinical Modification [ICD-9-CM] code 714.xx) who had a written prescription or order for their first injectable (self-injectable or infusible) bDMARD (etanercept, infliximab, adalimumab, certolizumab pegol, golimumab, anakinra, tocilizumab, abatacept, rituximab) in EHR. Index was the date of the first written prescription or order for a bDMARD during the period 2007-2013.

Patients were excluded if they did not have continuous pharmacy coverage in their health care plan for $\geq 6$ months before and after index; had evidence of bDMARD therapy in EHR; had claims in the 6 months before index; had multiple prescriptions for bDMARDs at index; or had been hospitalized within 30 days of index.

\section{Assessments}

Treatment Cohorts and Patient Characteristics. Patients were categorized as filling a bDMARD prescription via a pharmacy or receiving a bDMARD administration in outpatient/inpatient settings in Optum pharmacy and medical claims (using Healthcare Common Procedure Coding System) if (a) administrative data showed the prescription was filled within 30 days of the index bDMARD prescription date (hereafter referred to as the early biologic filler cohort); (b) if administrative data showed the prescription was filled within 31-180 days of the index bDMARD prescription date (hereafter referred to as the late biologic filler cohort); or (c) administration data showed that the prescription was not filled at all within 180 days of the index bDMARD (hereafter referred to as the nonfiller cohort).

Patient baseline characteristics, including the claims-based index of RA severity ([CIRAS]: 0 [low]-10 [high]), ${ }^{22}$ prescribing patterns for RA, and patient-reported pain scores ( $0-10$ on provider-determined scales) from EHR were assessed across all patients. In addition to bDMARDs, csDMARD prescribing patterns included methotrexate, sulfasalazine, hydroxychloroquine (or chloroquine), and leflunomide, as well as immunosuppressive agents (e.g., cyclosporine, tacrolimus, and azathioprine), gold compounds, and other related medications (e.g., minocycline, cyclophosphamide, penicillamine, and thalidomide). Change in pain scores ( $\geq 30$ days pre- and $\geq 30$ days post-index) were evaluated in the subgroup of patients with pain scores recorded in EHR.

Treatment Rationale. Humedica uses NLP to parse physician notes and identify the rationale behind prescribing decisions. This information is provided in the context of a drug action (e.g., switch, add, discontinue, titrate) and reason. The reasons for drug actions range from side effects (e.g., gastrointestinal intolerability) to access (e.g., product cost) and efficacy (e.g., lack or loss of efficacy). All information is derived from physicianwritten patient notes (i.e., not directly linked to the prescribing platform of EHR). Treatment rationale for stopping (early biologic fillers and late biologic fillers) or not filling (nonfillers) medication was assessed in the subgroup of patients for whom a reason was recorded in the physician-written patient notes.

Health Care Resource Use and Costs. Six-month, post-index, all-cause, and RA-related (any visit with ICD-9-CM code 714.xx or DMARD medication) costs, health care resource use (HCRU), and out-of-pocket costs, as identified within claims, were assessed in patients with both continuous medical and pharmacy coverage. Post-index total RA costs were adjusted using a generalized linear model that assumed gamma-distributed data and used a log link. As limited information has been published on primary nonadherence with bDMARDs in RA, independent variables were identified a priori for inclusion based on clinical expertise and included demographics, such as age at date of bDMARD prescription, sex, race, and Census Bureau region. Variables also included potential indicators of risk/severity, such as baseline CIRAS score, death, number of pre-index prescriptions, use of TNFi or non-TNFi bDMARDs, and any use of csDMARDs (pre- or post-index). Other variables were potential access barriers, including average household income imputed based on 3-digit ZIP code and insurance (e.g., health maintenance organization, preferred provider organization, point of service, exclusive provider organization, single payer, other) and commercial or Medicare Advantage; potential indicators of aversion to injectable medications with use of outpatient injectable medications during 180 days pre-index; and post-index classification as an early biologic filler, late biologic filler, or nonfiller.

The model was fit in 2 steps. In the first step, each of the independent variables was assessed by having it as the only variable in the model; if the $P$ value was $\leq 0.20$, it was kept and the final model was fit, including all such independent variables. In the second step (final model), 1 observation was excluded due to a missing value in the baseline RA severity score. Otherwise, statistical significance was declared at $P \leq 0.05$. The point estimates and corresponding standard errors (SEs) were inversetransformed from the log scale for reporting here. 


\section{TABLE 1 Cohort Selection}

\begin{tabular}{|c|c|c|}
\hline Patient Evaluation Group & $\mathrm{N}$ & $\%$ \\
\hline $\begin{array}{l}\text { 1. Total number of patients with temporal dataset overlap (2007-2013) in both the Humedica Clinical data and } \\
\text { Optum Claims data }\end{array}$ & $3,676,274$ & 100.0 \\
\hline 2. and with $\geq 1$ inpatient or $\geq 2$ outpatient visits with an RA diagnosis (2007-2013) in both EHR and claims & 43,949 & 1.2 \\
\hline 3. and with any injectable biologic prescription (2007-2013) in EHR & 5,652 & 0.2 \\
\hline 4. and excluding patients with $\geq 1$ type of injectable biologic DMARD on the same index date in EHR & 5,630 & 0.2 \\
\hline 5. and aged $\geq 18$ years & 5,593 & 0.2 \\
\hline 6. and with $\geq 6$ months of continuous enrollment pre-and post-index in claims & 1,990 & 0.05 \\
\hline 7. and without any claim/administration during 6 months pre-index in both EHR and claims & 1,125 & 0.03 \\
\hline 8. and without any inpatient hospitalization at index or during 30 days post-index in both EHR and claims & 1,076 & 0.03 \\
\hline 9. and with both medical and pharmacy coverage in claims during 6-month pre- and post-index periods & 375 & 0.01 \\
\hline 10. and excluding any patients receiving tofacitinib & 373 & 0.01 \\
\hline
\end{tabular}

DMARD = disease-modifying antirheumatic drug; EHR = Electronic Health Record

\section{Results}

\section{Patients}

In total, 373 patients were included in the analysis (Table 1). Of these, 170 (45.6\%) were categorized as early biologic fillers, 59 (15.8\%) as late biologic fillers, and 144 (38.6\%) as nonfillers.

Patient baseline characteristics are presented in Table 2. Most patients were white $(79.6 \%)$ and female $(73.5 \%)$, with a mean (standard deviation [SD]) age of 57.1 (14.7) years. Most patientshad commercial health care plans (60.6\%) and were most frequently located in the southern states (42.9\%). During the 6 months pre-index, the mean (SD) pain score among 33 patients with a reported value was 4.9 (3.2), mean (SD) CIRAS score was 4.0 (1.8), and mean (SD) number of monthly prescriptions for any reason was 1.6 (1.8) in EHR and 3.1 (3.0) in claims.

Compared with late biologic fillers and nonfillers, early biologic fillers were younger and more likely to be female with higher pain and RA severity scores pre-index, and they filled more prescriptions for any reason pre-index (Table 2). Compared with nonfillers, a greater proportion of early biologic fillers and late biologic fillers had commercial health care plans and were located in the southern states (Table 2).

\section{Index bDMARD Prescriptions}

A summary of index bDMARD prescriptions is presented in Table 3. In total, $88.7 \%$ of bDMARD prescriptions were for TNFi and 11.3\% were for non-TNFi bDMARDs. A greater proportion of nonfillers (13.9\%) had an index non-TNFi bDMARD prescription compared with early biologic fillers (9.4\%) and late biologic fillers (10.2\%). A greater proportion of early biologic fillers (71.2\%) had a csDMARD in the 180 days pre-index compared with late biologic fillers (64.4\%) and nonfillers (39.6\%).

\section{Treatment Patterns}

The majority of early biologic fillers (97.1\%) and late biologic fillers (81.4\%) filled or received a prescription that matched the index TNFi or non-TNFi prescription. Among early biologic fillers, 2.4\% (TNFi index) and 0.6\% (non-TNFi index) filled or received a prescription that did not match the index prescription. A small proportion of early biologic fillers (12.9\%) did not fill or receive a bDMARD during days 31-180 post-index (Table 3). Among late biologic fillers, $18.6 \%$ of patients who had a written prescription or order for an index TNFi filled or received a bDMARD that did not match the index medication. By contrast, all late biologic fillers who had a written prescription or order for an index non-TNFi bDMARD filled or received a matching prescription.

Prescriptions for csDMARDs were filled by $57.9 \%$ of patients during days -1 to 180 pre-index; $36.2 \%$ of patients during days 0 to 30 post-index; and $50.1 \%$ of patients during days 31 to 180 post-index (Table 3). During days 0 to 30 post-index, csDMARD use was greatest for early biologic fillers (45.9\%) and lowest for nonfillers (24.3\%); however, during days 31 to 180 post-index, csDMARD use was greatest for late biologic fillers (61.0\%) and lowest for nonfillers (35.4\%).

\section{Rationale for Stopping/Not Filling Medication}

Patient reasons for stopping medication (early biologic and late biologic fillers) or not filling medication (nonfillers) were available for 47 patients and are presented in Table 4. Most patients who stopped their index bDMARD did not have a reason recorded. Among those who had a reason recorded, side effects was most common, followed by efficacy and cost.

\section{Pain}

Only 23 of 373 (6.2\%) patients had pre- and post-index pain scores. The mean (SD) change in pain score was -1.6 (3.6) for early biologic fillers ( $\mathrm{n}=11 / 170 ; 6.5 \%), 0.6$ (5.5) for late biologic fillers $(\mathrm{n}=5 / 59 ; 8.5 \%)$, and 1.1 (3.2) for nonfillers $(\mathrm{n}=7 / 144 ; 4.9 \%)$.

\section{Health Care Resource Use}

Among 373 patients with pharmacy and medical coverage for 180 days post-index, the 6-month, unadjusted mean (SD), all-cause total costs were $\$ 18,260(\$ 11,284)$ for early biologic 
Primary Nonadherence, Associated Clinical Outcomes, and Health Care Resource Use Among Patients with Rheumatoid Arthritis Prescribed Treatment with Injectable Biologic Disease-Modifying Antirheumatic Drugs

TABLE 2 Patient Baseline Characteristics by bDMARD Fill Status

\begin{tabular}{|c|c|c|c|c|c|c|c|c|c|c|}
\hline \multirow[b]{2}{*}{ Age in years, mean (SD) } & \multicolumn{2}{|c|}{$\begin{array}{l}\text { All Patients } \\
\quad(\mathrm{N}=373)\end{array}$} & \multicolumn{2}{|c|}{$\begin{array}{l}\text { Early Biologic } \\
\text { Fillers }^{\mathrm{a}} \\
(\mathrm{n}=170)\end{array}$} & \multicolumn{2}{|c|}{$\begin{array}{l}\text { Late Biologic } \\
\text { Fillers }^{\mathrm{b}} \\
(\mathbf{n}=59)\end{array}$} & \multicolumn{2}{|c|}{$\begin{array}{l}\text { Nonfillersc } \\
(n=144)\end{array}$} & \multicolumn{2}{|c|}{$\begin{array}{l}\text { Late Biologic } \\
\text { Fillers and } \\
\text { Nonfillers } \\
\text { Combined } \\
(n=203)\end{array}$} \\
\hline & 57.1 & $(14.7)$ & 51.6 & $(13.4)$ & 55.9 & $(15.7)$ & 64.0 & $(12.8)$ & 61.7 & $(14.1)$ \\
\hline \multicolumn{11}{|l|}{ Sex, n (\%) } \\
\hline Female & 274 & $(73.5)$ & 128 & $(75.3)$ & 40 & $(67.8)$ & 106 & $(73.6)$ & 146 & $(71.9)$ \\
\hline \multicolumn{11}{|l|}{ Race, n (\%) } \\
\hline African American & 15 & $(4.0)$ & 9 & $(5.3)$ & 2 & $(3.4)$ & 4 & $(2.8)$ & 6 & $(3.0)$ \\
\hline Asian & 4 & $(1.1)$ & 2 & $(1.2)$ & 0 & & 2 & (1.4) & 2 & $(1.0)$ \\
\hline White & 297 & $(79.6)$ & 131 & $(77.1)$ & 48 & $(81.4)$ & 118 & $(81.9)$ & 166 & $(81.8)$ \\
\hline Other/unknown & 57 & $(15.3)$ & 28 & $(16.5)$ & 9 & $(15.3)$ & 20 & $(13.9)$ & 29 & $(14.3)$ \\
\hline \multicolumn{11}{|l|}{ Census Bureau region, n (\%) } \\
\hline Northeast & 29 & $(7.8)$ & 19 & (11.2) & 2 & (3.4) & 8 & (5.6) & 10 & (4.9) \\
\hline Midwest & 101 & $(27.1)$ & 33 & $(19.4)$ & 18 & $(30.5)$ & 50 & $(34.7)$ & 68 & $(33.5)$ \\
\hline South & 160 & $(42.9)$ & 85 & $(50.0)$ & 30 & $(50.9)$ & 45 & $(31.3)$ & 75 & $(37.0)$ \\
\hline West & 76 & $(20.4)$ & 30 & $(17.7)$ & 8 & $(13.6)$ & 38 & $(26.4)$ & 46 & $(22.7)$ \\
\hline Other/unknown & 7 & (1.9) & 3 & $(1.8)$ & 1 & $(1.7)$ & 3 & $(2.1)$ & 4 & $(2.0)$ \\
\hline Household income, mean (SD) & 44,545 & $(12,370)$ & 45,689 & $(14,342)$ & 42,286 & $(9,430)$ & 44,119 & $(10,722)$ & 43,585 & $(10,372)$ \\
\hline \multicolumn{11}{|l|}{ Business type on index date, $\mathrm{n}(\%)$} \\
\hline Commercial & 226 & $(60.6)$ & 140 & $(82.4)$ & 38 & $(64.4)$ & 48 & (33.3) & 86 & $(42.4)$ \\
\hline Medicare & 146 & $(39.1)$ & 30 & $(17.7)$ & 21 & $(35.6)$ & 95 & $(66.0)$ & 116 & $(57.1)$ \\
\hline Medicaid & 1 & $(0.3)$ & 0 & & 0 & & 1 & $(0.7)$ & 1 & $(0.5)$ \\
\hline \multicolumn{11}{|l|}{ Product type on index date, $\mathrm{n}(\%)$} \\
\hline Health maintenance organization & 125 & $(33.5)$ & 40 & $(23.5)$ & 14 & $(23.7)$ & 71 & $(49.3)$ & 85 & $(41.9)$ \\
\hline Preferred provider organization & 24 & $(6.4)$ & 11 & $(6.5)$ & 2 & $(3.4)$ & 11 & $(7.6)$ & 13 & $(6.4)$ \\
\hline Exclusive provider organization & 29 & $(7.8)$ & 17 & $(10.0)$ & 5 & $(8.5)$ & 7 & $(4.9)$ & 12 & $(5.9)$ \\
\hline Indemnity & 6 & $(1.6)$ & 2 & $(1.2)$ & 2 & $(3.4)$ & 2 & $(1.4)$ & 4 & $(2.0)$ \\
\hline Point of service & 140 & $(37.5)$ & 88 & $(51.8)$ & 27 & $(45.8)$ & 25 & $(17.4)$ & 52 & $(25.6)$ \\
\hline Other & 49 & $(13.1)$ & 12 & $(7.1)$ & 9 & (15.3) & 28 & $(19.4)$ & 37 & $(18.2)$ \\
\hline Pain score during 6 months pre-index, $\mathrm{n}(\%)$ & 33 & $(8.9)$ & 16 & $(9.4)$ & 7 & $(11.9)$ & 10 & $(6.9)$ & 17 & $(8.4)$ \\
\hline Pain score, mean (SD) & 4.9 & $(3.2)$ & 6.3 & $(2.2)$ & 4.4 & $(3.5)$ & 2.9 & $(3.4)$ & 3.5 & $(3.4)$ \\
\hline RA-severity score during 6 months pre-index, mean (SD) & 4.0 & $(1.8)$ & 4.7 & $(1.7)$ & 4.4 & $(1.6)$ & 3.2 & $(1.5)$ & 3.5 & $(1.6)$ \\
\hline \multicolumn{11}{|c|}{ Number of monthly prescriptions during 6 months pre-index, mean (SD) } \\
\hline Humedica (EHR) & 1.6 & $(1.8)$ & 1.7 & $(2.0)$ & 1.5 & $(1.6)$ & 1.4 & $(1.8)$ & 1.4 & $(1.7)$ \\
\hline Optum (claims) & 3.1 & $(3.0)$ & 3.9 & $(3.1)$ & 3.5 & $(2.7)$ & 2.1 & $(2.8)$ & 2.5 & $(2.8)$ \\
\hline $\begin{array}{l}\text { Number of rheumatology visits during } 6 \text { months } \\
\text { pre-index, mean (SD) }\end{array}$ & & $(2.0)$ & 1.2 & $(2.0)$ & 1.5 & $(2.4)$ & 0.8 & (1.6) & 1.0 & (1.9) \\
\hline
\end{tabular}

abDMARD prescription filled within 30 days of index $b D M A R D$.

bDDMARD prescription filled within 31-180 days of index $b D M A R D$.

cbDMARD prescription not filled at all within 180 days of index $b D M A R D$.

bDMARD = biologic disease-modifying antirheumatic drug; EHR=Electronic Health Record; $R A=$ rheumatoid arthritis; $S D=$ standard deviation.

fillers versus $\$ 13,113(\$ 6,653)$ for late biologic fillers and $\$ 6,334(\$ 9,850)$ for nonfillers. Six-month, unadjusted mean (SD), total RA-related costs were highest for early biologic fillers $(\$ 12,896[\$ 6,194])$ versus late biologic fillers $(\$ 8,386$ $[\$ 4,222])$ and nonfillers $(\$ 1,555[\$ 4,863]$; Figure 1). Most of the unadjusted mean total RA-related costs were attributed to bDMARDs for early biologic fillers (74.7\%) and late biologic fillers (71.8\%), and outpatient visits for nonfillers (85.8\%). RA-related pharmacy costs accounted for $84.6 \%$ of the unadjusted mean total RA-related cost difference between early biologic fillers and late biologic fillers and nonfillers combined. In the regression model, 6-month, adjusted mean (SE), total RA-related costs were $\$ 12,467(\$ 1,843)$ for early biologic fillers versus $\$ 9,631(\$ 1,805)$ for late biologic fillers and $\$ 1,577$ (\$236) for nonfillers. The unadjusted 6-month mean (SD) total patient out-of-pocket cost for the index bDMARD was $\$ 777$ (\$1,097) for early biologic fillers versus $\$ 652$ (\$996) for late biologic fillers. Early biologic fillers had greater RA-related pharmacy and medical resource use versus late biologic fillers and nonfillers combined (Figure 2). 
Primary Nonadherence, Associated Clinical Outcomes, and Health Care Resource Use Among Patients with Rheumatoid Arthritis Prescribed Treatment with Injectable Biologic Disease-Modifying Antirheumatic Drugs

TABLE 3 bDMARD and csDMARD Treatment Patterns by bDMARD Fill Status

\begin{tabular}{|c|c|c|c|c|c|c|c|c|c|c|}
\hline & & $\begin{array}{l}\text { tients } \\
373)\end{array}$ & Biolo & $\begin{array}{l}\text { rly } \\
\text { Fillers } \\
\text { 170) }\end{array}$ & Biolo & $\begin{array}{l}\text { ate } \\
\text { Fillers } \\
\end{array}$ & & $\begin{array}{l}\text { illers }^{c} \\
144)\end{array}$ & $\begin{array}{r}\text { Late Biol } \\
\text { Nonfille }\end{array}$ & $\begin{array}{l}\text { Fillers and } \\
\text { ombined } \\
\text { 3) }\end{array}$ \\
\hline Index bDMARD, n (\%) & & & & & & & & & & \\
\hline TNFi & 331 & $(88.7)$ & 154 & $(90.6)$ & 53 & $(89.8)$ & 124 & $(86.1)$ & 177 & $(87.2)$ \\
\hline Non-TNFi biologic & 42 & $(11.3)$ & 16 & $(9.4)$ & 6 & $(10.2)$ & 20 & $(13.9)$ & 26 & $(12.8)$ \\
\hline csDMARD use, $\mathrm{n}(\%)$ & & & & & & & & & & \\
\hline Any & 235 & $(63.0)$ & 128 & (75.3) & 45 & (76.3) & 62 & (43.1) & 107 & $(52.7)$ \\
\hline Days -1 to 180 pre-index & 216 & $(57.9)$ & 121 & $(71.2)$ & 38 & $(64.4)$ & 57 & $(39.6)$ & 95 & $(46.8)$ \\
\hline Days 0 to 30 post-index & 135 & $(36.2)$ & 78 & $(45.9)$ & 22 & (37.3) & 35 & $(24.3)$ & 57 & $(28.1)$ \\
\hline Days 31 to 180 post-index & 187 & $(50.1)$ & 100 & $(58.8)$ & 36 & $(61.0)$ & 51 & $(35.4)$ & 87 & $(42.9)$ \\
\hline Any injectable biologic DMA & days & 180 po & $\mathrm{x}, \mathrm{n}(\%$ & & & & & & & \\
\hline Yes & 207 & $(55.5)$ & 148 & $(87.1)$ & 59 & $(100.0)$ & 0 & & 59 & $(29.1)$ \\
\hline No & 166 & $(44.5)$ & 22 & (12.9) & 0 & & 144 & $(100.0)$ & 144 & $(70.9)$ \\
\hline
\end{tabular}

abDMARD prescription filled within 30 days of index $b D M A R D$.

bDDMARD prescription filled within 31-180 days of index $b D M A R D$.

cDDMARD prescription not filled at all within 180 days of index $b D M A R D$.

bDMARD = biologic disease-modifying antirheumatic drug; csDMARD = conventional synthetic disease-modifying antirheumatic drug; TNFi= tumor necrosis factor inhibitor .

\section{Discussion}

A major challenge among patients with chronic medical conditions, such as RA, is optimizing patient adherence to therapy, as the implications of nonadherence can be far-reaching in terms of reducing treatment effectiveness and increasing health care costs. Before the advent of EHR and electronic prescribing, it was difficult to identify rates of primary nonadherence to prescribed medications; however, advances in the use of EHR and claims databases have provided new insights into the magnitude of this problem. Here, we used EHR and claims data to evaluate the filling of newly prescribed initial bDMARDs for the treatment of RA and to characterize patient outcomes in a real-world setting.

The results reported here are in agreement with previous outpatient studies suggesting that primary nonadherence is a significant issue among patients with RA..$^{9,15,23,24}$ Our data indicated that more than half $(54.4 \%)$ of patients who were prescribed initial bDMARDs, most of which were TNFi, did not fill the prescription or receive a bDMARD within 30 days of the index prescription; by 180 days post-index, more than one-third (38.6\%) of patients had not yet filled or received a bDMARD. Additional analyses indicated that $56.9 \%$ of nonfillers did not have a csDMARD in the pre- and post-index periods. This raises the question of whether nonfillers proceed long-term without the benefits of an effective DMARD to halt disease progression. Another important factor is whether these patients self-medicate with over-the-counter, nonsteroidal antiinflammatory drugs, prescription selective cyclooxygenase 2 (COX-2) inhibitors, or opioid analgesics, each of which has its own risks.

To determine the reasons for primary nonadherence, we evaluated treatment rationale information extracted via NLP.
Although the reasons for not initiating treatment or switching treatment were rarely documented in EHR, several reasons may be considered, including patient out-of-pocket costs, particularly for the Medicare population. Late biologic fillers and nonfillers were more likely to have Medicare coverage compared with early fillers. Previous research has suggested that patient out-of-pocket costs are significantly associated with bDMARD abandonment in Medicare patients with RA. ${ }^{25}$ Although not evaluated in this study, an understanding of the impact of the coverage gap in Medicare patients may also be warranted. Hopson et al. (2014) found that patients meeting the catastrophic coverage limit and using a specialty pharmacy had a decreased likelihood of abandoning bDMARD therapy. ${ }^{25}$ Furthermore, in our study, almost $20 \%$ of late biologic fillers had filled or received a bDMARD medication that was different from the index TNFi prescription, perhaps suggesting time for coverage decisions (e.g., out-of-pocket costs, selfadministration vs. outpatient injection/infusion, insurance company "steps") that may have resulted in the need to switch treatments or receive bDMARDs in a different setting. This may be particularly important in considering low-income subsidy and dual-eligible patients who were not included in the study by Hopson et al. and cannot be easily identified in the current study database.

Patient preference for noninjectable treatments may be another reason, as supported by research in a commercial population that suggests route of administration is the most important factor for patients when they consider whether to initiate bDMARDs. ${ }^{26}$ Interestingly, late biologic fillers had the lowest proportion of patients with pre-index injectable medications, followed by nonfillers. Patient preference may also be affected by disease severity, as suggested by the fact that early 
Primary Nonadherence, Associated Clinical Outcomes, and Health Care Resource Use Among Patients with Rheumatoid Arthritis Prescribed Treatment with Injectable Biologic Disease-Modifying Antirheumatic Drugs

\begin{tabular}{|c|c|c|c|c|}
\hline \multirow[b]{3}{*}{ Any reason, n (\%) } & \multicolumn{4}{|c|}{$\begin{array}{l}\text { Rationale for Stopping (Early Biologic } \\
\text { Fillers and Late Biologic Fillers) and } \\
\text { Not Filling (Nonfillers) bDMARDs }\end{array}$} \\
\hline & $\begin{array}{l}\text { All Patients } \\
(\mathrm{N}=373)\end{array}$ & $\begin{array}{l}\text { Early } \\
\text { Biologic } \\
\text { Fillersa } \\
(\mathbf{n}=170)\end{array}$ & $\begin{array}{c}\text { Late Biologic } \\
\text { Fillers } \\
(\mathbf{n}=59)\end{array}$ & $\begin{array}{c}\text { Nonfillers }^{\mathrm{c}} \\
(\mathrm{n}=144)\end{array}$ \\
\hline & $47 \quad(12.6)$ & $14 \quad(8.2)$ & $12(20.3)$ & $21 \quad(14.6)$ \\
\hline Cost & $4 \quad(8.5)$ & (7.1) & $1 \quad(8.3)$ & $2 \quad(9.5)$ \\
\hline Efficacy & $6 \quad(12.8)$ & $2(14.3)$ & $2 \quad(16.7)$ & $(9.5)$ \\
\hline Side effects & $10(21.3)$ & 3 (21.4) & $3(25.0)$ & $4 \quad(19.0)$ \\
\hline No reason given & $39(83.0)$ & $12 \quad(85.7)$ & $8 \quad(66.7)$ & $19(90.5)$ \\
\hline \multicolumn{5}{|c|}{$\begin{array}{l}\text { abDMARD prescription filled within } 30 \text { days of index } b D M A R D . \\
\text { bbDMARD prescription filled within } 31-180 \text { days of index } b D M A R D . \\
\text { cbDMARD prescription not filled at all within } 180 \text { days of index } b D M A R D . \\
\text { bDMARD = biologic disease-modifying antirheumatic drug. }\end{array}$} \\
\hline
\end{tabular}

biologic fillers had the highest disease severity and pain scores and were most likely to have received prior csDMARD therapy versus late biologic fillers and nonfillers. Furthermore, fear of needles may also play a role in patient primary nonadherence. In a recent research survey conducted to assess the preference of patients with RA $(\mathrm{N}=310)$ for a needle-free device versus a needle-based device, $45 \%$ of patients reported a "needle phobia." ${ }^{27}$ In this analysis, however, only $2.1 \%$ of physicians $(\mathrm{N}=373)$ reported a fear of needles in their patients.

Several studies have used U.S. prescription claims data to specifically evaluate adherence to bDMARDs among patients with RA. ${ }^{16-19}$ However, in contrast with the analysis reported here that evaluated primary nonadherence, all of the studies evaluated secondary nonadherence. Although this study did not evaluate secondary nonadherence, $12.9 \%$ of early fillers did not have a bDMARD fill or administration during days 31-180. Furthermore, although the concurrent use of csDMARDs has been shown to have a beneficial effect on bDMARD adherence and clinical outcomes, more than $40 \%$ of early biologic fillers did not receive a csDMARD during days 31-180 post-index. Another important limitation of the studies is that they did not evaluate the relationship between adherence and clinical response. Consequently, EULAR recently recommended that the questions, "How good is patient adherence to biologic agents, and can a lack of adherence be related to loss of efficacy?" be included in a future research agenda. ${ }^{5}$

Few studies have evaluated economic outcomes associated with nonadherence to bDMARDs in patients with RA. However, the findings reported to date in secondary nonadherence studies suggest that although greater pharmacy costs are the main driver of total health care costs among adherent patients in the 1-year period after treatment initiation, nonadherent patients incur greater HCRU, including inpatient and outpatient visits as well as laboratory services. ${ }^{16,28}$ By contrast, in this primary nonadherence study, HCRU was found to be

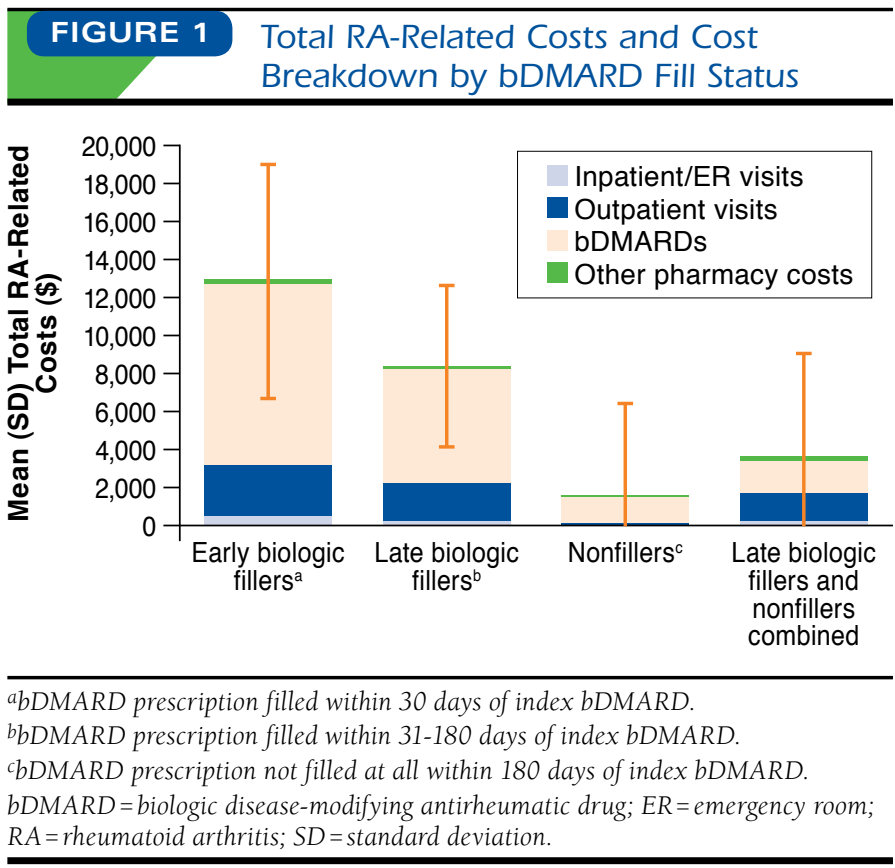

greater for adherent patients than for nonadherent patients during short-term observation. This is likely attributed to the short (6-month) observation window, as one source of direct medical costs is the need for hospitalizations for orthopedic surgery or nursing home admissions, which may occur much later in the course of disease, although geographic variation is also common. ${ }^{29}$ Furthermore, this study was not designed to evaluate indirect costs attributed to lost productivity. Indirect costs can exceed direct medical costs often by a factor of 3-4. ${ }^{30}$ Treatment with bDMARDs, such as TNFi, has been shown to be associated with a cost per quality-adjusted life year $<\$ 50,000$ (cost-effective), without accounting for the impact on indirect costs. ${ }^{29}$

\section{Limitations}

The analysis reported here provides insight into adherence to bDMARDs based on a small group of retrospectively identified patients with RA; however, like all EHR- and claims-based analyses, several limitations must be considered.

Although patients were selected based on the presence of an RA diagnosis, it is possible that the bDMARDs were prescribed for other reasons. Because of concerns regarding the limited size of the patient population with integrated data, and given that the analysis focused on primary nonadherence, which can occur irrespective of the diagnosis, no additional exclusion of other conditions for which bDMARDs may be prescribed or administered was implemented. Similarly, a 6-month preand post-index period was selected for evaluation and may have resulted in misclassification of bDMARD experience and 


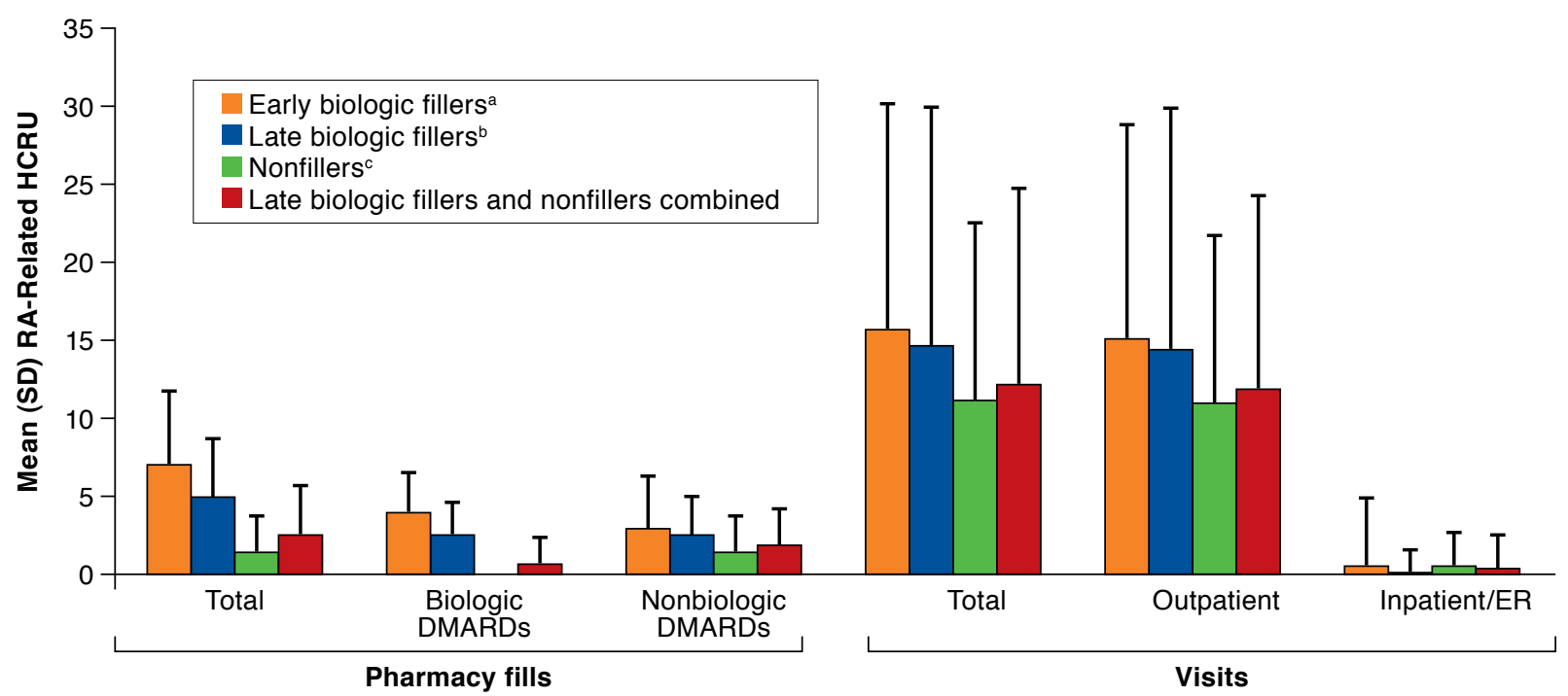

abDMARD prescription filled within 30 days of index $b D M A R D$.

bDDMARD prescription filled within 31-180 days of index $b D M A R D$.

cbDMARD prescription not filled at all within 180 days of index $b D M A R D$.

DDMARD = biologic disease-modifying antirheumatic drug; DMARD = disease-modifying antirheumatic drug; ER=emergency room; HCRU =health care resource use; $R A=$ rheumatoid arthritis; $S D=$ standard deviation .

limited evaluation of the longer-term consequences of primary nonadherence. However, requiring $\geq 12$ months of observation in EHR decreased the initial population by approximately two-thirds, upon which patients required overlapping claims enrollment, further substantially reducing the remaining cohort. Thus, extending the pre- and post-index periods is warranted in future analyses of a larger population.

EHR data are not intended for research use and are limited by the physician's challenges in using complex and timeconsuming EHR platforms while balancing large volumes of patients and providing ample time for patient care. Thus, key data are often missing, particularly multidimensional clinical measures of severity or outcomes. In particular, availability of at least 2 pain scores was limited to only $6 \%$ of the study cohort; thus, generalizability of the change in pain score findings is not possible in the current study and serves only as hypothesis-generating to support future research efforts. Similarly, reasons for discontinuation or not filling a bDMARD were found in approximately $13 \%$ of patients and are not generalizable to other patients or settings. This further highlights the challenges of using existing EHR, particularly physician notes. Advanced methods such as NLP can assist in overcoming the time and costs of manual chart abstraction but cannot address a limited yield due to underreporting. Physician incentives, such as Meaningful Use and pay for performance, are expected to improve documentation in EHR for selected measures per disease. In addition, the ability to link claims, EHR, and other data sources in the future will help to address current gaps.

Claims data are collected for payment processing; therefore, a claim does not indicate that the patient took the medication. A period of no claim may not indicate nonadherence but may be due to temporary discontinuation of therapy because of illness. Coding errors could also affect the ability to detect all relevant HCRU. Regarding costs, the charged or requested amount billed by the provider was used as the cost in this analysis, although this value may be highly variable, since it often has little relationship to the actual amount paid.

\section{Conclusions}

These findings demonstrate that among the RA patient cohort studied, overall adherence to bDMARDs is suboptimal, and primary nonadherence may be occurring at a rate not recognized by clinicians. Treatment adherence assessments before and after regular follow-up visits could enable practicing clinicians to manage patients effectively, and importantly, reduce rates of primary nonadherence. Furthermore, there is a need to implement strategies to assess treatment efficacy to optimize quality and control costs. ACR currently recommends 6 disease activity measures, including the Clinical Disease Activity Index, Disease Activity Score with 28-joint counts 
(erythrocyte sedimentation rate or C-reactive protein), Patient Activity Scale (PAS), PAS-II, Routine Assessment of Patient Index Data with 3 measures, and Simplified Disease Activity Index. ${ }^{31}$ Future research should focus on population-level, prospectively collected cohorts to evaluate the long-term consequences of undertreatment on clinical and economic outcomes.

\section{Authors}

JAMES HARNETT, PharmD, MS, is Senior Director, Real World Data and Analytics, and DANIEL WIEDERKEHR, BS, is Director, Worldwide Policy, Pfizer, New York, New York. ROBERT GERBER, PharmD, is Senior Director, Outcomes and Evidence, and DAVID GRUBEN, PhD, is Senior Director, Statistics, Pfizer, Groton, Connecticut. JEFFREY BOURRET, PharmD, is Senior Director, North America Medical Affairs, and ANDREW KOENIG, DO, is Senior Director, North America Medical Affairs, Pfizer, Collegeville, Pennsylvania.

AUTHOR CORRESPONDENCE: James Harnett, PharmD, MS, Pfizer, 235 East 42nd St., New York, NY 10017.

Tel.: 212.733.3753; E-mail: James.Harnett@pfizer.com.
5. Smolen JS, Landewé R, Breedveld FC, et al. EULAR recommendations for the management of rheumatoid arthritis with synthetic and biological disease-modifying antirheumatic drugs: 2013 update. Ann Rheum Dis. 2014;73(3):492-509

6. Koncz T, Pentek M, Brodszky V, Ersek K, Orlewska E, Gulacsi L. Adherence to biologic DMARD therapies in rheumatoid arthritis. Expert Opin Biol Ther. 2010;10(9):1367-78.

7. Blum MA, Koo D, Doshi JA. Measurement and rates of persistence with and adherence to biologics for rheumatoid arthritis: a systematic review. Clin Ther. 2011;33(7):901-13.

8. World Health Organization. Adherence to long-term therapies: evidence for action. 2003. Available at: http://apps.who.int/iris/bitstream/10665/42682/1/9241545992.pdf. Accessed November 30, 2015.

9. Fischer MA, Stedman MR, Lii J, et al. Primary medication non-adherence: analysis of 195,930 electronic prescriptions. J Gen Intern Med. 2010;25(4):284-90.

10. Benjamin RM. Medication adherence: helping patients take their medicines as directed. Public Health Rep. 2012;127(1):2-3.

11. IMS Institute for Healthcare Informatics. Avoidable costs in U.S. healthcare. The $\$ 200$ billion opportunity from using medicines more responsibly. 2013. Available at: https://www.imshealth.com/en/thought-leadership/ webinar-library/avoidable-costs-in-us-healthcare-200-billion-opportunity. Accessed November 30, 2015.

12. Grijalva CG, Chung CP, Arbogast PG, Stein CM, Mitchel EF Jr., Griffin MR. Assessment of adherence to and persistence on disease-modifying antirheumatic drugs (DMARDs) in patients with rheumatoid arthritis. Med Care. 2007:45(10 Suppl 2):S66-76.

13. Salt E, Frazier SK. Adherence to disease-modifying antirheumatic drugs in patients with rheumatoid arthritis: a narrative review of the literature. Orthop Nurs. 2010;29(4):260-75.

14. Horne R, Chapman SC, Parham R, Freemantle N, Forbes A, Cooper V. Understanding patients' adherence-related beliefs about medicines prescribed for long-term conditions: a meta-analytic review of the NecessityConcerns Framework. PLoS One. 2013;8(12):e80633.

15. Fallis BA, Dhalla IA, Klemensberg J, Bell CM. Primary medication nonadherence after discharge from a general internal medicine service. PLoS One. 2013;8(5):e61735.

16. Borah BJ, Huang X, Zarotsky V, Globe D. Trends in RA patients' adherence to subcutaneous anti-TNF therapies and costs. Curr Med Res Opin. 2009;25(6):1365-77.

17. Li P, Blum MA, Von Feldt J, Hennessy S, Doshi JA. Adherence, discontinuation, and switching of biologic therapies in Medicaid enrollees with rheumatoid arthritis. Value Health. 2010;13(6):805-12.

18. Harley CR, Frytak JR, Tandon N. Treatment compliance and dosage administration among rheumatoid arthritis patients receiving infliximab etanercept, or methotrexate. Am J Manag Care. 2003;9(6 Suppl):S136-43.

19. Curkendall S, Patel V, Gleeson M, Campbell RS, Zagari M, Dubois R. Compliance with biologic therapies for rheumatoid arthritis: do patient outof-pocket payments matter? Arthritis Rheum. 2008;59(10):1519-26.

20. U.S. Department of Health \& Human Services. Guidance regarding methods for de-identification of protected health information in accordance with the Health Insurance Portability and Accountability Act (HIPAA) Privacy Rule. 2012. Available at: http://www.hhs.gov/ocr/privacy/hipaa/ understanding/coveredentities/De-identification/guidance.html. Accessed November 30, 2015

21. Public welfare. Other requirements relating to uses and disclosures of protected health information. 45 CFR \$164.514(b). April 23, 2015. Available at: http://www.ecfr.gov/cgi-bin/retrieveECFR?gp=1\&SID=e0170bc87bl0624 763295b7800ce770e\&ty=HTML\&h=L\&mc=true\&r=PART\&n=pt45.1.164\# se45.1.164_1514. Accessed November 30, 2015. 
22. Ting G, Schneeweiss S, Scranton R, et al. Development of a health care utilisation data-based index for rheumatoid arthritis severity: a preliminary study. Arthritis Res Ther. 2008;10(4):R95.

23. Raebel MA, Carroll NM, Ellis JL, Schroeder EB, Bayliss EA. Importance of including early nonadherence in estimations of medication adherence. Ann Pharmacother. 2011;45(9):1053-60.

24. Fischer MA, Choudhry NK, Brill G, et al. Trouble getting started: predictors of primary medication nonadherence. Am J Med. 2011;124(11):1081.e9-22.

25. Hopson S, Saverno KR, Liu LZ, AL-Sabbagh A, Orazem J, Pasquale MK. Impact of out-of-pocket costs on initial and subsequent prescription fills among new initiators of biologic therapies indicated for rheumatoid arthritis. Poster presented at: Academy of Managed Care Pharmacy 2014 Nexus; October 7-10, 2014; Boston, MA. Available at: http://www.amcp.org/ WorkArea/DownloadAsset.aspx?id=18543.

26. Louder A, Singh A, Cappelleri J, Aten A, Koenig A, Pasquale M. Understanding patient preferences associated with the use of therapies for rheumatoid arthritis: results of a conjoint analysis. Arthritis \& Rheumatism. 2014;66(11):S1043.
27. Bennison C, Chansler M, Hibbard J, Turanin J. Adoption, adherence and preferences regarding injectable drug delivery by patients with rheumatoid arthritis: results of a research study. Poster presented at: AAPS National Biotechnology Conference, May 20-22; 2013; San Diego, CA. Available at: http://www.zogenix.com/content/technology/documents/ AAPSPosterAdoptionofSelfInjectRxBennisonMay2013.pdf.

28. Tang B, Rahman M, Waters HC, Callegari P. Treatment persistence with adalimumab, etanercept, or infliximab in combination with methotrexate and the effects on health care costs in patients with rheumatoid arthritis. Clin Ther. 2008;30(7):1375-84.

29. Kavanaugh A. Economic consequences of established rheumatoid arthritis and its treatment. Best Pract Res Clin Rheumatol. 2007;21(5):929-42.

30. Pincus $T$. The underestimated long term medical and economic consequences of rheumatoid arthritis. Drugs. 1995;(50 Suppl):11-14.

31. Anderson J, Caplan L, Yazdany J, et al. Rheumatoid arthritis disease activity measures: American College of Rheumatology recommendations for use in clinical practice. Arthritis Care Res (Hoboken). 2012;64(5):640-47. 